\title{
Prognostic significance of preoperative prognostic nutritional index in colorectal cancer: results from a retrospective cohort study and a meta-analysis
}

\author{
Yuchong Yang ${ }^{1, *}$, Peng Gao ${ }^{1, *}$, Xiaowan Chen ${ }^{1}$, Yongxi Song ${ }^{1}$, Jinxin Shi ${ }^{1}$, Junhua

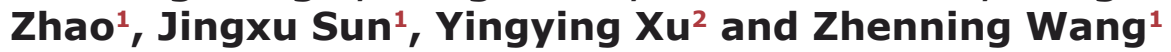 \\ ${ }^{1}$ Department of Surgical Oncology and General Surgery, First Hospital of China Medical University, Shenyang City, PR China \\ 2 Department of Breast Surgery, First Hospital of China Medical University, Shenyang City, PR China \\ * These authors have contributed equally to this work \\ Correspondence to: Zhenning Wang, email: josieon826@sina.cn \\ Keywords: colorectal cancer, meta-analysis, prognostic nutritional index, prognosis, TNM staging \\ Received: April 29, $2016 \quad$ Accepted: June 07, $2016 \quad$ Published: June 17, 2016
}

\section{ABSTRACT}

The preoperative prognostic nutritional index (PNI) may forecast colorectal cancer (CRC) outcomes, but the evidence is not conclusive. Here, we retrospectively analyzed a cohort of patients from the Department of Surgical Oncology at the First Hospital of China Medical University (CMU-SO). We also conducted a meta-analysis of eleven cohort studies. Bayesian Information Criterion (BIC) was used to determine the optimal PNI cut-off values for classifying prognosis in the patients from the CMU-SO. The result from CMU-SO and meta-analysis both confirmed that low PNI was significantly associated with a poor prognosis and advanced TNM stages. Among the patients from the CMU-SO, the optimal cut-off values were "41-45-58" (PNI < 41, $41 \leq$ PNI < 45, $45 \leq$ PNI < 58, PNI $\geq 58$ ), which divided patients into 4 stages. The BIC value for TNM staging combined with the PNI was smaller than that of TNM staging alone (-325.76 vs. -310.80$)$. In conclusion, low PNI was predictive of a poor prognosis and was associated with clinicopathological features in patients with CRC, and the 41-45-58 four-stage division may be suitable for determining prognosis. PNI may thus provide an additional index for use along with the current TNM staging system to determine more accurate CRC prognoses.

\section{INTRODUCTION}

Colorectal cancer (CRC) is the second most common cancer in women and third most common in men; an estimated 1.4 million cases and 693,900 deaths occurred in 2012 [1]. Despite advancements in surgery, adjuvant chemoradiotherapy and targeted therapy $[2,3]$, the prognosis of CRC patients remains unsatisfactory. At present, TNM staging is considered the primary prognostic indicator. However, TNM staging is limited because patients with the same stage may have different clinical outcomes. Therefore, a new more accurate prognostic indicator for CRC patients is required to improve prognostic accuracy.

Some studies have reported that the progression and prognosis of cancer are determined not only by tumor features but also by nutritional and immunological conditions [4-7]. The prognostic nutritional index (PNI), calculated from serum albumin levels and peripheral lymphocyte count, reflects both the nutritional and immune status of the patient $[8,9]$. Many recent studies demonstrate that PNI is a significant prognostic indicator for some malignancies [10], including hepatocellular carcinoma [11], pancreatic cancer [12], laryngeal cancer [13], renal cell carcinoma [14], and gastric carcinoma [15]. Although several studies on PNI have evaluated CRC prognosis $[16,17]$, few pooled studies and few studies with large sample size have explored the prognostic role of PNI in patients with CRC. Moreover, to the best of our knowledge, no studies have focused on the use of PNI 
as an additional index on the basis of the current TNM staging system. On the other hand, controversy still exists concerning the optimal cut-off values and how groups should be classified by PNI staging to determine CRC prognosis.

In this study, we retrospectively analyzed a cohort of patients from the Department of Surgical Oncology at the First Hospital of China Medical University (CMU-SO). We also conducted a meta-analysis with eleven cohort studies. In addition, we aimed to identify the optimal cutoff values and the most suitable divisions by PNI staging for determining prognosis in patients with CRC.

\section{RESULTS}

Most relevant studies divide PNI into two groups to study its prognostic value. We split PNI into two groups according to the BIC method, allowing the comparison of the results from the meta-analysis. In patients from the CMU-SO, the median PNI was 51.3 (range, 32.3-71.2). We calculated Bayesian information criterion (BIC) values using different cut-off values; when the cut-off value was set at 45 , the BIC values were the smallest for overall survival (OS) and cause-specific survival (CSS) (-9.527 and -8.427 , respectively), indicating that the optimal cutoff value for splitting into two groups was 45 . Of the 2062 patients evaluated, 275 patients (13.34\%) with a PNI $<45$ and 1787 patients $(86.66 \%)$ with a PNI $\geq 45$ were classified into the low PNI and high PNI groups, respectively.

\section{PNI and clinicopathological features}

For patients from the CMU-SO, low PNI was significantly associated with older age, larger tumor size, tumor location in the colon, poor differentiation, increased tumor depth, advanced TNM stages, and fewer patients on postoperative chemotherapy. There was no significant difference in the sexes $(P=0.193)$, lymph node metastasis status $(P=0.276)$, or distant metastasis status $(P=0.072)$ between the low- and high-PNI groups (Table 1).

For patients from the meta-analysis, there were significantly more patients with older age, positive lymph node metastasis, advanced TNM stages, postoperative complications, and who did not receive postoperative chemotherapy in the low-PNI group than in the high-PNI group. The differences in tumor depth $(P=0.172)$ and tumor differentiation $(P=0.131)$ were not significant between the two groups (Table S1).

\section{PNI and prognosis}

In the patients from the CMU-SO, the 5-year OS rate was $67.4 \%$ in the low-PNI group and $77.8 \%$ in the
high-PNI group ( $P<0.001$, Figure 1A, Table 2$)$. The 5 -year CSS rate was $69.5 \%$ in the low-PNI group and $80.3 \%$ in the high-PNI group $(P<0.001$, Figure $1 \mathrm{~B}$, Table $2)$. Furthermore, subgroup analysis shows the relationship of PNI with patient prognosis at each stage. OS was significantly different between the two groups at stages II and III $(P<0.001, P=0.046)$ but not at stages I and IV $(P=0.101, P=0.757$, Figure 2$)$. CSS was significantly different between the groups at stages I and II $(P=0.002$, $P<0.001)$ but not at stages III and IV $(P=0.058, P=$ 0.841 , Figure 2). In addition, Cox multivariate analysis indicates that low PNI was a poor independent prognostic factor for $\mathrm{OS}(\mathrm{HR}=1.282,95 \% \mathrm{CI}=1.020-1.610, P=$ $0.033)$ and $\mathrm{CSS}(\mathrm{HR}=1.343,95 \% \mathrm{CI}=1.051-1.715, P=$ 0.018 , Table 2).

In the patients from the meta-analysis, the pooled result indicates that low PNI was significantly associated with poor $\mathrm{OS}(\mathrm{HR}=1.972,95 \% \mathrm{CI}=1.536-2.532, P<$ 0.001 , Figure $3 \mathrm{~A})$ and $\mathrm{CSS}(\mathrm{HR}=1.479,95 \% \mathrm{CI}=1.185$ $1.846, P=0.001$, Figure 3B). Further subgroup analysis indicates that the prognostic value of PNI for OS was not undermined by subgroup analysis on the basis of geographical region, surgery, TNM stage, cut-off value, sample size, or study quality (Table S2).

\section{Optimal cut-off values}

To date, the optimal PNI cut-off values and the division of groups by PNI staging remain unknown. In this study, we calculated BIC values for different cut-off values and a different number of stage divisions (from two to five divisions). Our results indicate that $\mathrm{BIC}$ values from the 41-45-58 four-stage division (PNI $<41,41 \leq$ PNI $<45,45$ $\leq \mathrm{PNI}<58$, and $\mathrm{PNI} \geq 58$ ) were the smallest for OS and CSS (Figure 4, Table S3). The Kaplan-Meier curves for different stage divisions, which indicate the smallest BIC values, are shown in Figure 5.

\section{Evaluation of the prognostic capacity of TNM staging combined with PNI}

We calculated the BIC values of TNM staging combined with PNI and of TNM staging alone. The BIC value of TNM staging combined with the 41-45-58 fourstage division was smaller than that of TNM staging alone $(-325.76$ vs. -310.80$)$.

\section{DISCUSSION}

We retrospectively analyzed a large sample of patients from the CMU-SO. Low PNI was an independent prognostic factor for poor OS and CSS and was significantly associated with advanced tumor features such as older age, larger tumor size, and advanced TNM stages. 
Table 1: Associations of PNI status with clinicopathological features in CRC patients from CMU-SO

\begin{tabular}{|c|c|c|c|c|}
\hline & & PNI status & & \\
\hline Variable & Number (\%) & High PNI (\%) & Low PNI (\%) & $P$ \\
\hline Sample size & $2062(100)$ & 1787 (86.7) & 275 (13.3) & \\
\hline Age, Mean \pm SD, y & & $60.7 \pm 10.5$ & $65.0 \pm 10.0$ & $<0.001$ \\
\hline Gender & & & & 0.193 \\
\hline Male & $1166(56.5)$ & $997(55.8)$ & $169(61.5)$ & \\
\hline Female & $896(43.5)$ & $790(44.2)$ & $106(38.5)$ & \\
\hline Tumor size $(\mathrm{cm})$ & & & & $<0.001$ \\
\hline$\geq 4.6$ & $1035(50.2)$ & $952(53.3)$ & $200(72.7)$ & \\
\hline$<4.6$ & $1027(49.8)$ & $835(46.7)$ & $75(27.3)$ & \\
\hline Tumor location & & & & $<0.001$ \\
\hline Colon & $871(42.2)$ & 701 (39.2) & $170(61.8)$ & \\
\hline Rectum & $1191(57.8)$ & $1086(60.8)$ & $105(38.2)$ & \\
\hline Differentiation & & & & 0.002 \\
\hline Well - moderate & 1885 (91.4) & $1648(92.2)$ & $237(86.2)$ & \\
\hline Poor - undifferentiated & $177(8.6)$ & $139(7.8)$ & $38(13.8)$ & \\
\hline pT category & & & & 0.001 \\
\hline $\mathrm{T} 1$ & $58(2.8)$ & $54(3.0)$ & $4(1.6)$ & \\
\hline $\mathrm{T} 2$ & $381(18.5)$ & $352(19.7)$ & $29(10.5)$ & \\
\hline $\mathrm{T} 3$ & $852(41.3)$ & $722(40.4)$ & $130(47.3)$ & \\
\hline $\mathrm{T} 4$ & $771(37.4)$ & $659(36.9)$ & $112(40.7)$ & \\
\hline pN category & & & & 0.276 \\
\hline pN0 & $1216(59.0)$ & $1058(59.2)$ & $158(57.5)$ & \\
\hline $\mathrm{pN} 1$ & $619(30.0)$ & $540(30.2)$ & 79 (28.7) & \\
\hline $\mathrm{pN} 2$ & $227(11.0)$ & $189(10.6)$ & $38(13.8)$ & \\
\hline Distant metastasis & & & & 0.072 \\
\hline Negative & $2018(97.9)$ & $1753(98.1)$ & $265(96.4)$ & \\
\hline Positive & $44(2.1)$ & $34(1.9)$ & $10(3.6)$ & \\
\hline TNM stage & & & & 0.001 \\
\hline $\mathrm{I}$ & $351(17.0)$ & $326(18.2)$ & $25(9.1)$ & \\
\hline II & $855(41.5)$ & $726(40.6)$ & $129(46.9)$ & \\
\hline III & $812(39.4)$ & $701(39.2)$ & $111(40.4)$ & \\
\hline IV & $44(2.1)$ & $34(1.9)$ & $10(3.6)$ & \\
\hline Postoperative chemotherapy & & & & $<0.001$ \\
\hline Absent & $886(43.0)$ & $732(41.0)$ & $154(56.0)$ & \\
\hline Present & $1176(57.0)$ & $1055(59.0)$ & $121(44.0)$ & \\
\hline
\end{tabular}

Abbreviations, PNI: prognostic nutritional index; SD: standard deviation.

In addition, we retrospectively evaluated the patients from the eleven studies included in the meta-analysis. To the best of our knowledge, this meta-analysis is the first study to systematically assess the prognostic value of PNI and the association between PNI and clinicopathological features in patients with CRC. The meta-analysis results were similar to those of the CMU-SO study, demonstrating that low PNI was significantly associated with poor outcomes and advanced tumor features in CRC.

There are several possible explanations for the 
Table 2: Univariate and multivariate survival analyses of OS and CSS in CRC patients from CMU-SO

\begin{tabular}{|c|c|c|c|c|c|c|c|c|}
\hline \multirow[b]{3}{*}{ Variable } & \multicolumn{4}{|l|}{ Overall survival } & \multicolumn{4}{|c|}{ Cancer-Specific Survival } \\
\hline & \multicolumn{2}{|l|}{ Univariate } & \multicolumn{2}{|l|}{ Multivariate } & \multicolumn{2}{|l|}{ Univariate } & \multicolumn{2}{|l|}{ Multivariate } \\
\hline & HR $(95 \%$ CI) & $P$ & HR $(95 \%$ CI $)$ & $P$ & HR $(95 \%$ CI $)$ & $P$ & HR $(95 \%$ CI) & $P$ \\
\hline \multicolumn{9}{|l|}{ Gender } \\
\hline Female & 1 & & & & 1 & & & \\
\hline Male & $\begin{array}{|ll|}1.224 \\
1.469)\end{array}$ & 0.030 & & & $\begin{array}{l}1.158 \\
1.408)\end{array}$ & 0.141 & & \\
\hline \multicolumn{9}{|l|}{ Age (y) } \\
\hline$\geq 62$ & 1 & & 1 & & 1 & & & \\
\hline$<62$ & $\begin{array}{|ll|}0.761 & (0.636- \\
0.911) & \\
\end{array}$ & 0.003 & $\begin{array}{ll}0.778 & (0.645- \\
0.938) & \\
\end{array}$ & 0.009 & $\begin{array}{ll}0.877 & (0.723- \\
1.062) & \\
\end{array}$ & 0.179 & & \\
\hline \multicolumn{9}{|l|}{ Tumor Size $(\mathrm{cm})$} \\
\hline$\geq 4.6$ & 1 & & & & 1 & & & \\
\hline$<4.6$ & $\begin{array}{|ll|}0.894 & (0.748- \\
1.068) & \\
\end{array}$ & 0.217 & & & $\begin{array}{|ll|}0.888 & (0.733- \\
1.076) & \\
\end{array}$ & 0.224 & & \\
\hline \multicolumn{9}{|l|}{ Tumor location } \\
\hline Colon & 1 & & & & 1 & & & \\
\hline Rectum & $\begin{array}{|ll|}1.078 & (0.899- \\
1.292) & \\
\end{array}$ & 0.418 & & & $\begin{array}{ll}1.104 & (0.908- \\
1.343) & \\
\end{array}$ & 0.321 & & \\
\hline \multicolumn{9}{|l|}{ Differentiation } \\
\hline Well - moderate & 1 & & 1 & & 1 & & 1 & \\
\hline $\begin{array}{l}\text { Poor } \\
\text { undifferentiated }\end{array}$ & $\begin{array}{ll}2.252 \\
2.906)\end{array}$ & $<0.001$ & $\begin{array}{l}1.574 \quad(1.213- \\
2.041)\end{array}$ & 0.001 & $\begin{array}{l}2.317 \\
3.041)\end{array}$ & $<0.001$ & $\begin{array}{l}.532(1.162- \\
2.021)\end{array}$ & 0.003 \\
\hline \multicolumn{9}{|l|}{ pT category } \\
\hline T1 & 1 & & 1 & & 1 & & 1 & \\
\hline $\mathrm{T} 2$ & \begin{tabular}{|l|}
2.548 \\
$8.168)$
\end{tabular} & 0.116 & $\begin{array}{ll}2.404 \\
7.715)\end{array}$ & 0.140 & $\begin{array}{|ll|}1.833 \\
5.952)\end{array}$ & 0.313 & $\begin{array}{l}1.699(0.522- \\
5.523)\end{array}$ & 0.378 \\
\hline T3 & $\begin{array}{l}5.321 \\
16.623)\end{array}$ & 0.004 & $\begin{array}{ll}3.098 & (0.987- \\
9.719) & \\
\end{array}$ & 0.053 & $\begin{array}{l}4.500 \\
14.076)\end{array}$ & 0.010 & $\begin{array}{l}2.445(0.777- \\
7.690)\end{array}$ & 0.126 \\
\hline $\mathrm{T} 4$ & \begin{tabular}{|l|}
7.645 \\
$23.919)$
\end{tabular} & $<0.001$ & $\begin{array}{l}4.507 \\
14.189)\end{array}$ & 0.010 & $\begin{array}{l}6.779 \\
21.224)\end{array}$ & 0.001 & $\begin{array}{l}3.659(1.160- \\
11.540) \\
\end{array}$ & 0.027 \\
\hline \multicolumn{9}{|l|}{ pN category } \\
\hline $\mathrm{pN} 0$ & 1 & & 1 & & 1 & & 1 & \\
\hline $\mathrm{pN} 1$ & $\begin{array}{|ll|}4.355 & (3.492- \\
5.430) & \\
\end{array}$ & $<0.001$ & $\begin{array}{ll}4.299 & (3.426- \\
5.394) & \\
\end{array}$ & $<0.001$ & $\begin{array}{l}5.020 \\
6.434)\end{array}$ & $<0.001$ & $\begin{array}{l}4.812(3.731- \\
6.207) \\
\end{array}$ & $<0.001$ \\
\hline $\mathrm{pN} 2$ & \begin{tabular}{|l|}
10.349 \\
$13.246)$
\end{tabular} & $<0.001$ & $\begin{array}{l}9.954 \\
12.890)\end{array}$ & $<0.001$ & $\begin{array}{l}12.722 \text { (9.707- } \\
16.675)\end{array}$ & $<0.001$ & $\begin{array}{l}11.612(8.759- \\
15.394)\end{array}$ & $<0.001$ \\
\hline \multicolumn{9}{|l|}{$\begin{array}{l}\text { Distant } \\
\text { metastasis }\end{array}$} \\
\hline Negative & 1 & & 1 & & 1 & & 1 & \\
\hline Positive & $\begin{array}{|ll|}3.818 & (2.484- \\
5.867) & \\
\end{array}$ & $<0.001$ & $\begin{array}{ll}2.450 & (1.581- \\
3.797) & \\
\end{array}$ & $<0.001$ & $\begin{array}{|ll|}4.143 & (2.666- \\
6.437) & \\
\end{array}$ & $<0.001$ & $\begin{array}{l}2.362(1.511- \\
3.695)\end{array}$ & $<0.001$ \\
\hline \multicolumn{9}{|l|}{ TNM stage } \\
\hline $\mathrm{I}$ & 1 & & & & 1 & & & \\
\hline II & $\begin{array}{|ll|}1.876 & (1.190- \\
2.959) & \\
\end{array}$ & 0.007 & & & $\begin{array}{ll}2.598 \\
4.682)\end{array}$ & 0.001 & & \\
\hline III & $\begin{array}{l}8.791 \quad(5.761- \\
13.414)\end{array}$ & $<0.001$ & & & $\begin{array}{l}13.727(7.878- \\
23.918)\end{array}$ & $<0.001$ & & \\
\hline IV & \begin{tabular}{|l|}
16.109 \\
$28.975)$
\end{tabular} & $<0.001$ & & & $\begin{array}{l}26.243(13.112- \\
52.525)\end{array}$ & $<0.001$ & & \\
\hline \multicolumn{9}{|l|}{$\begin{array}{l}\text { Postoperative } \\
\text { chemotherapy }\end{array}$} \\
\hline Absent & 1 & & 1 & & 1 & & 1 & \\
\hline Present & $\begin{array}{|ll|}0.697 & (0.583- \\
0.834) & \\
\end{array}$ & $<0.001$ & $\begin{array}{ll}0.497 & (0.410- \\
0.602) & \\
\end{array}$ & $<0.001$ & $\begin{array}{|ll|}0.795 & (0.656- \\
0.963) & \\
\end{array}$ & 0.019 & $\begin{array}{l}0.522 \\
0.638)\end{array}(0.427-$ & $<0.001$ \\
\hline
\end{tabular}




\begin{tabular}{|c|c|c|c|c|c|c|c|c|}
\hline PNI & & & & & & & & \\
\hline$\geq 45$ & 1 & & 1 & & 1 & & 1 & \\
\hline$<45$ & $\begin{array}{l}1.648 \\
2.062)\end{array}$ & $<0.001$ & $\begin{array}{l}1.282 \\
1.610)\end{array}$ & 0.033 & $\begin{array}{l}1.680 \\
2.136)\end{array}$ & $<0.001$ & $\begin{array}{l}1.343(1.051- \\
1.715)\end{array}$ & 0.018 \\
\hline
\end{tabular}

Abbreviations, HR: hazard ratio; CI: confidence interval.
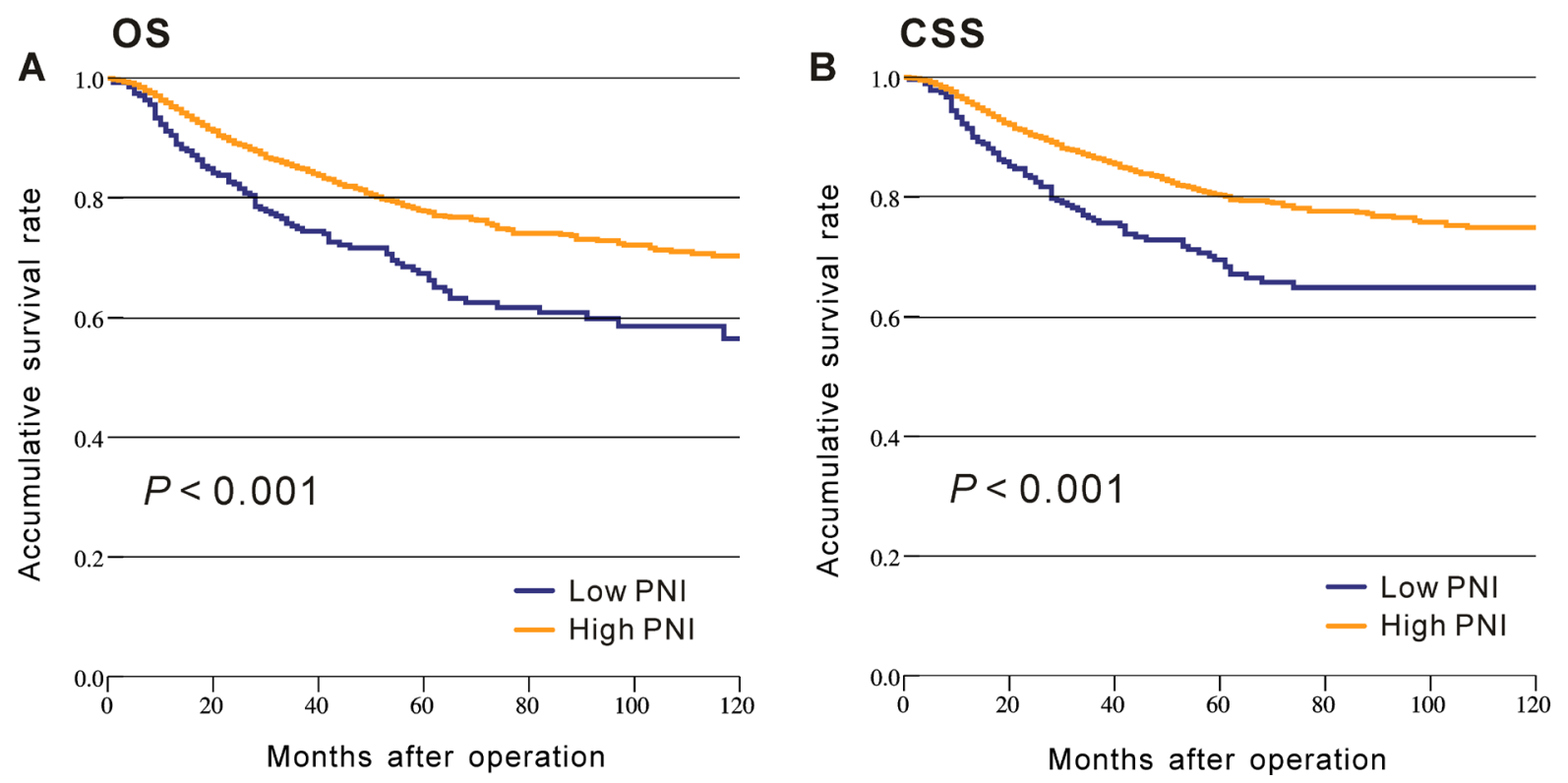

Figure 1: Kaplan-Meier analysis of survival based on low and high prognostic nutritional index among patients from CMU-SO: A. overall survival; B. cancer-specific survival.

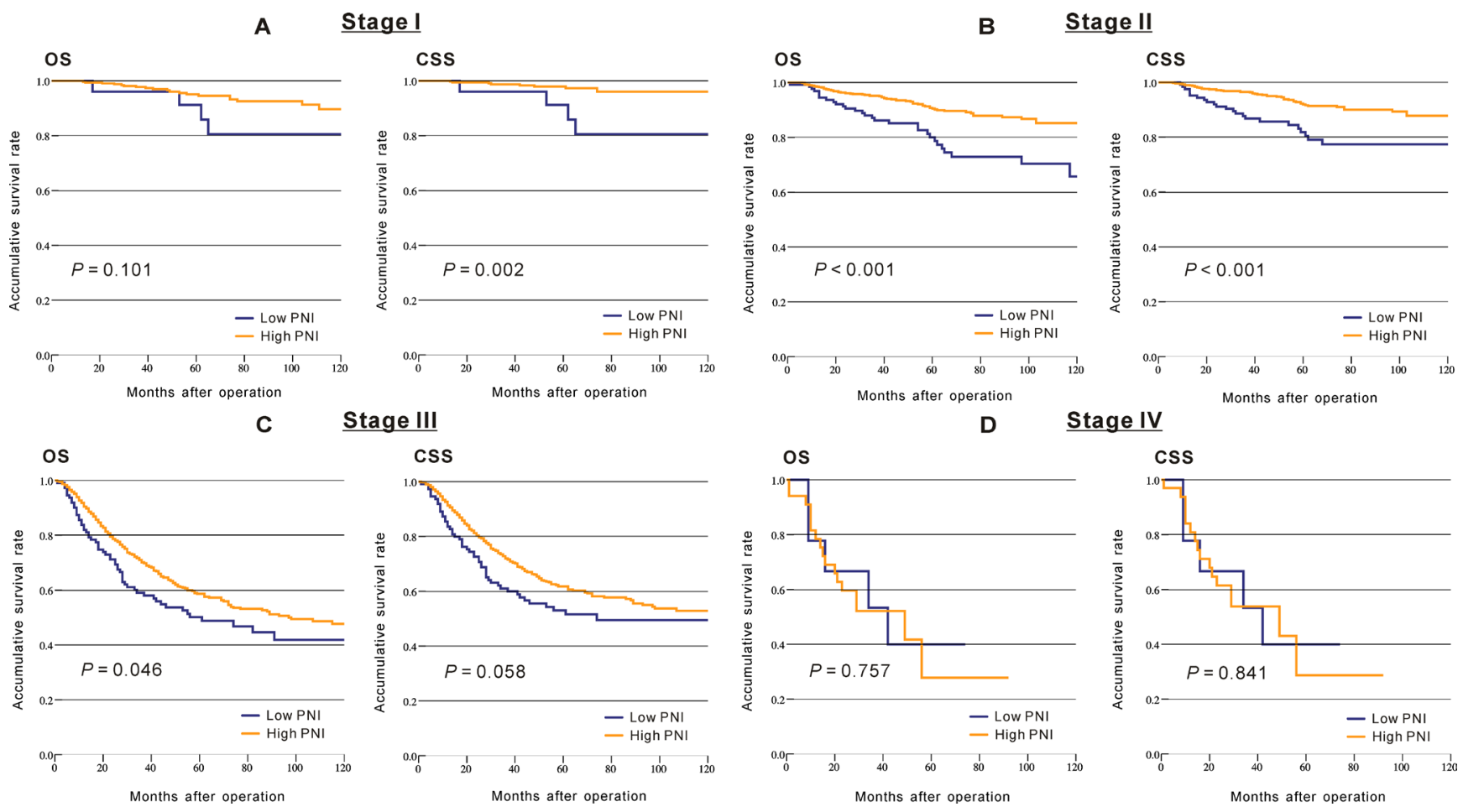

Figure 2: Kaplan-Meier analysis of the overall survival (OS) and cancer-specific survival (CSS) based on low and high prognostic nutritional index among patients from CMU-SO with stage I (A., OS, $P=0.101$; CSS, $P=0.002$ ), stage II (B., OS, $P<0.001$; CSS, $P<0.001$ ), stage III (C., OS, $P=0.046$; CSS, $P=0.058$ ) and stage IV (D., OS, $P=0.757$; CSS, $P=0.841$ ). 
association between low PNI and poor prognosis in CRC. First, lymphocytes and serum albumin are significantly associated with prognosis of cancer patients [7, 18, 19].
PNI reflects the nutritional and immune condition of patients because it is based on the peripheral lymphocyte count and serum albumin levels. For this reason alone, PNI

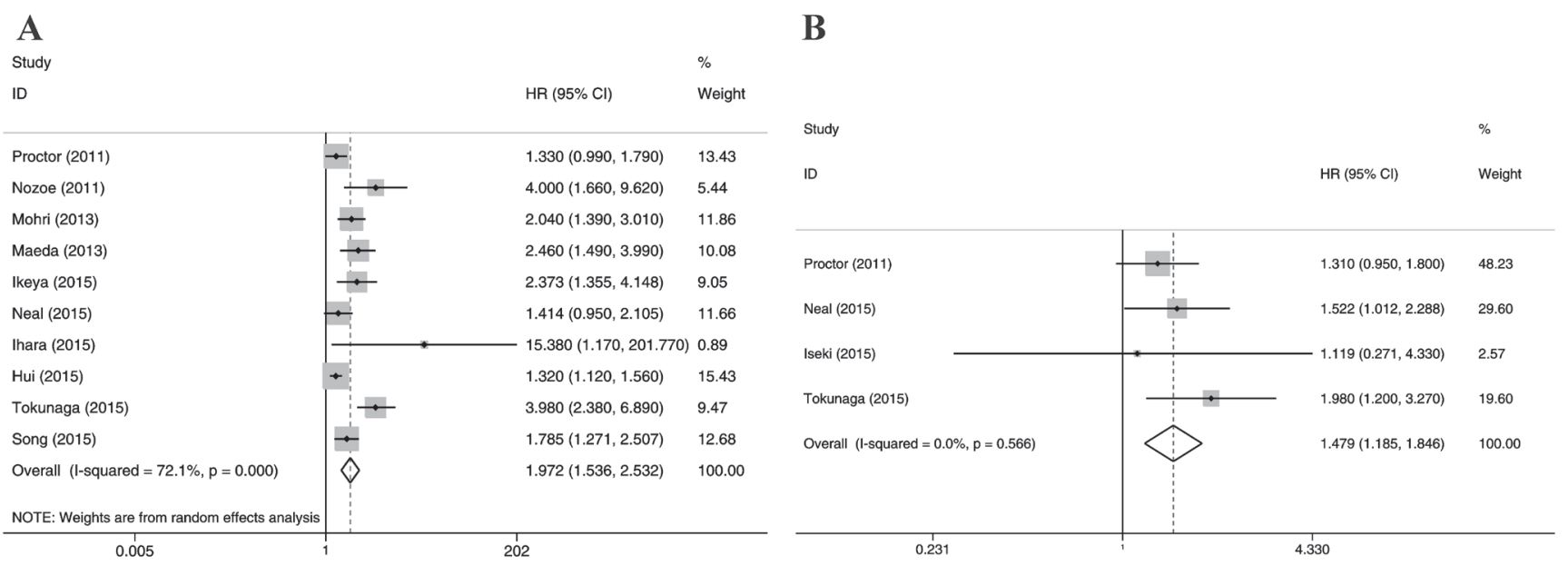

Figure 3: Meta-analysis of the association between low prognostic nutritional index and survival in CRC: A. overall survival; B. cancer-specific survival.

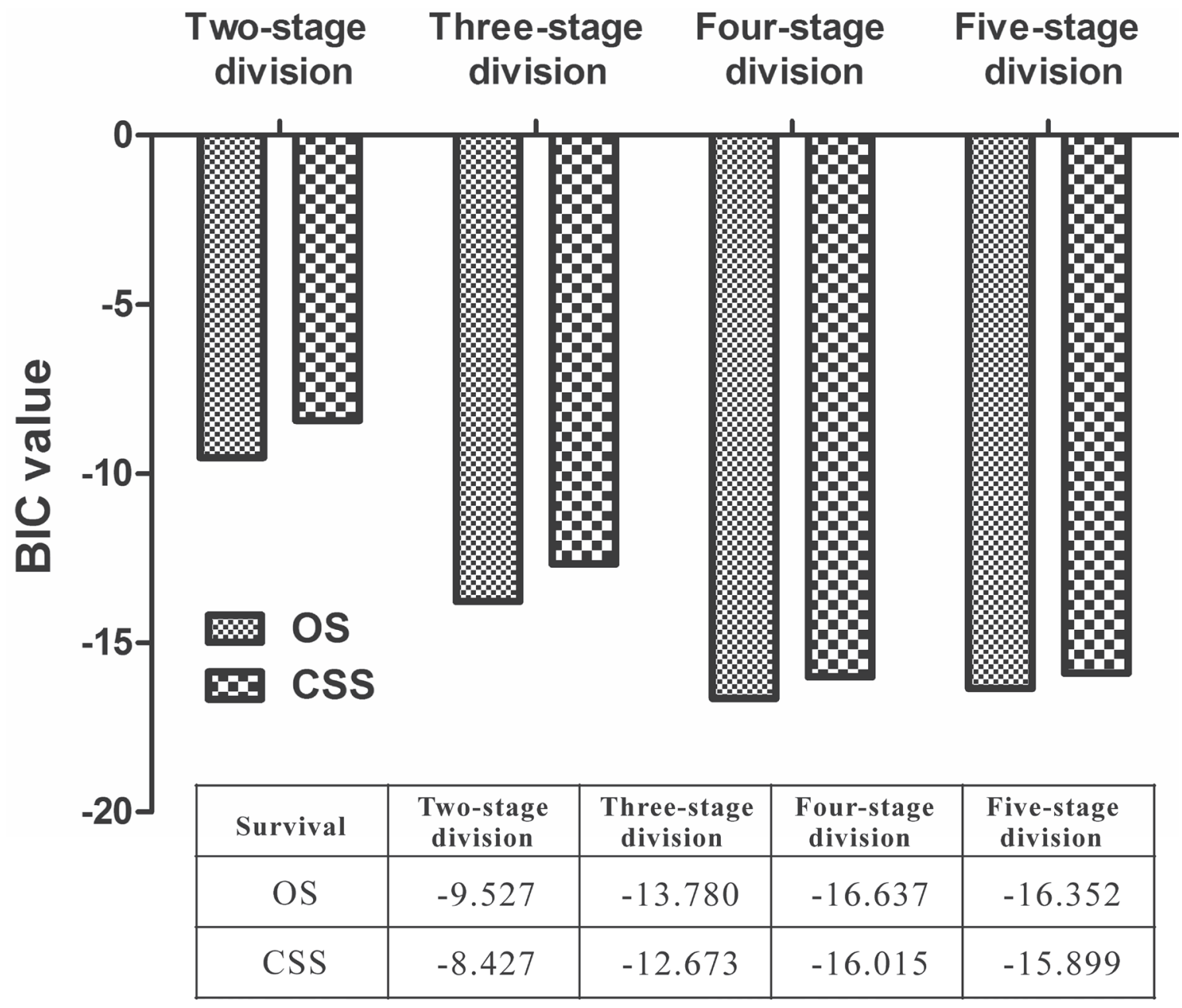

Figure 4: The smallest Bayesian Information Criterion values of overall survival and cancer-specific survival for a different number of stage divisions (from two to five divisions). 
correlates with the prognosis of cancer patients. Second, our results indicate that low PNI in CRC patients was significantly associated with advanced tumor features and occurrence of postoperative complications. The significant relationship between postoperative complications and poor prognosis of CRC was confirmed by other studies [20, 21]. Therefore, this relationship may partly explain the association between low PNI and poor survival of patients with CRC. Third, poor immune and nutritional status may lead to a delay in postoperative adjuvant therapy or even abandonment of treatment. Indeed, the results of the CMU-SO study and meta-analysis indicate that significantly more patients in the low-PNI group did not receive postoperative chemotherapy compared with those in the high-PNI group. However, whether PNI should influence the clinical decision for postoperative adjuvant therapy remains to be determined.

In the meta-analysis, the result of the subgroup analysis indicates that low PNI was significantly associated with poor OS at all TNM stages. However, for the patients from the CMU-SO study, OS was significantly different between the low-PNI and high-PNI groups at stages II and III but not at stages I and IV. CSS was significantly different between the groups at stages I and II but not at stages III and IV. We observed that low PNI tended to be associated with poor OS at stage I $(P=0.101)$ and with poor CSS at stage III $(P=0.058)$ and that there were only 44 patients at stage IV in the CMU-SO study. Large-scale studies are necessary to confirm this result.

In this meta-analysis, the PNI cut-off value for the included studies varied between 40.0 and 45.5 (median: 45.0). Kanda et al. [12] reported that a PNI value greater than or equal to 50 was regarded as normal, a value smaller than 50 indicated mild malnutrition, a value smaller than 45 indicated moderate to severe malnutrition, and a value smaller than 40 indicated serious malnutrition. On the other hand, a few studies $[15,22]$ used receiver operating characteristic (ROC) curve analysis, and another [17] used classification and regression tree analysis to identify the optimal cut-off value; Fu et al. [13] used the Cut off
A Three-stage division
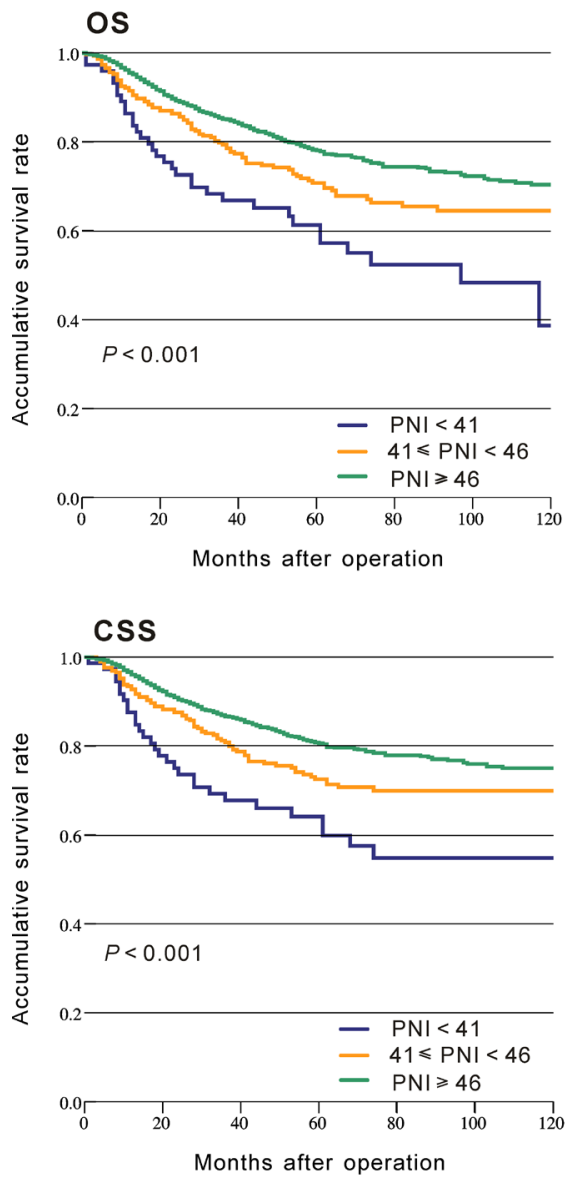

B Four-stage division
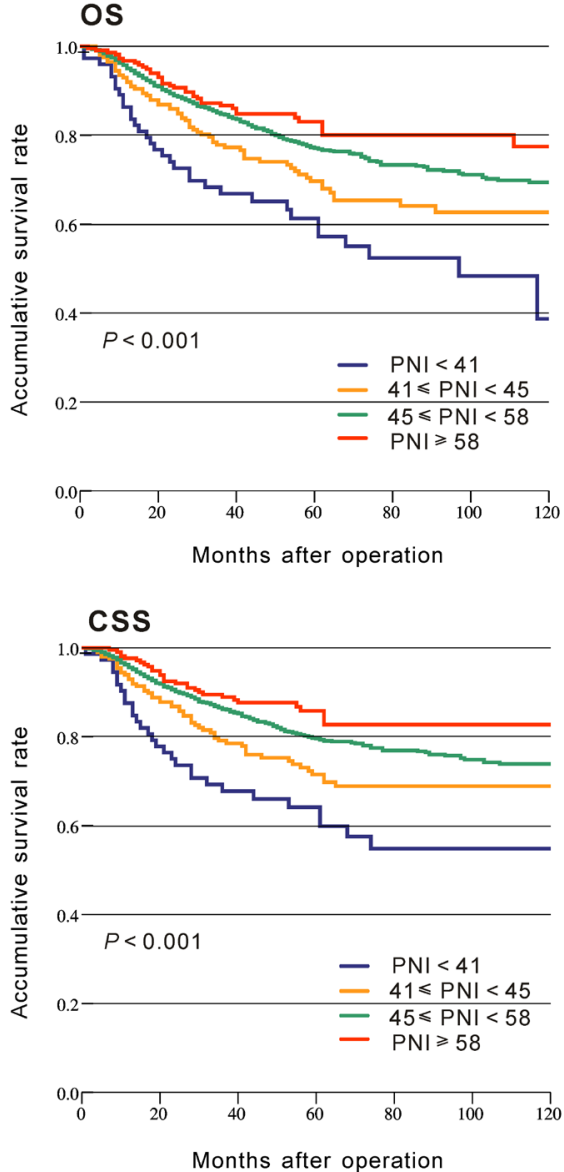

C Five-stage division
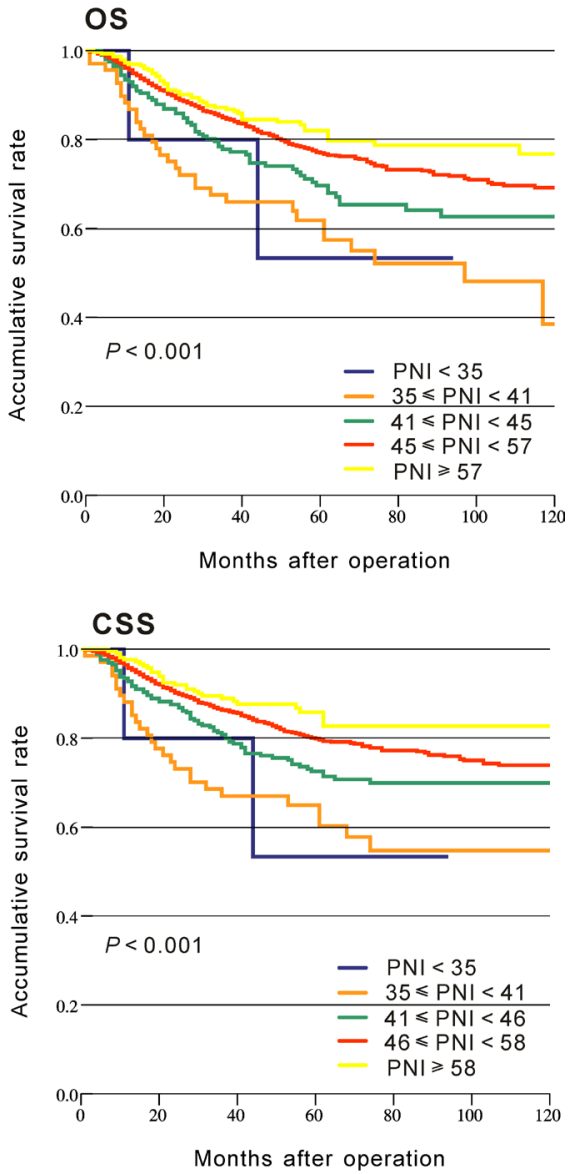

Figure 5: Kaplan-Meier analysis of the overall survival (OS) and cancer-specific survival (CSS) for a different number of stage divisions which indicated smallest Bayesian Information Criterion value (two-stage division see Figure 1). A. 41-46 three-stage division; B. 41-45-58 four-stage division; C. five-stage division (OS, 35-41-45-57 four-stage division; CSS, 35-41-46-58 four-stage division) 
Finder software to determine two cut-off values and divide patients into three groups. Therefore, the optimal cutoff value and the division of the groups by PNI staging remain unclear. In this study, BIC, which is one of most common methods for evaluating the predictive capacity of disease staging, was used to determine cut-off values and the number of stages. We calculated the BIC values for the cut-off values and stage divisions (from two to five divisions). The BIC value in the 41-45-58 four-stage division was the smallest and obviously lower than that of the two-stage and three-stage divisions. In addition, the increased number of divisions such as the five-stage division did not make the BIC value lower than that of the four-stage division and may result in increased complexity of staging. Therefore, we concluded that this fourstage division was the most useful system to determine prognosis for the patients from the CMU-SO. However, the cut-off values and stages identified by a single cohort may not apply to other independent cohorts. Therefore, these results need to be confirmed by future studies.

The BIC value for the combination of TNM staging and PNI in the 41-45-58 four-stage division was smaller than that of TNM staging alone, indicating that TNM combined with PNI in the four-stage division was a better division than TNM staging alone. Therefore, PNI complements the currently used TNM staging system and may increase the accuracy of prognosis for patients with CRC.

There were several limitations in our study. First, our dataset was collected retrospectively from a single institution and the studies included in the meta-analysis were retrospective. Second, all patients from the CMUSO study were Chinese and most patients from the metaanalysis were from Asian countries; whether the results of this study can be applied to other populations remains unknown.

In conclusion, low PNI was a poor prognostic indicator and was significantly associated with clinicopathological features in patients with CRC. The 4145-58 four-stage division may be a suitable PNI staging classification to determine prognosis of patients with CRC. PNI may serve as a supplementary index based on the current TNM staging system in CRC.

\section{MATERIALS AND METHODS}

\section{Patients from the CMU-SO}

Information on one cohort of patients with CRC who underwent primary tumor resection at the CMU-SO from March 1995 to May 2014 was collected retrospectively. The patients were selected on the basis of the following criteria: (1) CRC was based on pathological examination; (2) patients had not taken neoadjuvant therapy or anti- inflammatory medications before surgery; (3) laboratory data were obtained before surgery, and patients with synchronous or metachronous tumors were excluded. A total of 2,062 patients were included in this study. Followup was completed for all patients by September 2015. The median follow-up was 50 months (range of 1-185). Clinicopathological features, including age, sex, tumor size, tumor location, macroscopic type, differentiation grade, TNM stage, and preoperative laboratory data, were obtained from the medical records of the patients. PNI was calculated as $10 \times$ albumin level $(\mathrm{g} / \mathrm{dl})+0.005 \times$ total lymphocyte count (per $\mathrm{mm}^{3}$ ) $[8,9]$. The CRC stage was classified according to the seventh edition of the AJCC/ UICC TNM classification system.

\section{Literature search and meta-analysis}

We used the search terms "prognostic nutritional index" and "colon cancer/rectal cancer/colorectal cancer" to perform a literature search in PubMed, Embase, and Web of Science databases up to December 31, 2015. Eligible studies were selected on the basis of the following criteria: (1) diagnosis of CRC was based on histopathologic examination; (2) clinicopathological or/ and prognostic values of preoperative PNI in CRC were reported; (3) outcome measures were extracted directly or estimated from the studies indirectly; (4) PNI was calculated as $10 \times$ albumin level $(\mathrm{g} / \mathrm{dl})+0.005 \times$ total lymphocyte count (per $\mathrm{mm}^{3}$ ). Finally, we included 11 cohort studies $[16,17,23-31]$ comprising 3,788 patients in the meta-analysis (the flow diagram of the study selection procedure was shown in Figure S1). The median sample size was 219 patients (range of 80-1321). Seven studies were from Japan, two from the UK, one from China, and one from South Korea. The quality of these studies was assessed using the Newcastle-Ottawa quality assessment scale (NOS) [32]. NOS scores $\geq 6$ (median scores of the studies) were assigned as high-quality studies. The characteristics of the included studies are shown in Table S4.

\section{Statistical analysis}

Categorical variables are presented as absolute values and percentages and were compared via the chisquare test. Continuous data are expressed as the mean \pm standard deviation (SD) and compared using the MannWhitney U test. Survival rates, including overall survival (OS) and cancer-specific survival (CSS), were analyzed using the Kaplan-Meier method and compared using the log-rank test. Multivariate analysis was performed using Cox's proportional hazards model. We assessed the predictive capacity of different stage divisions by measuring discrimination, which is the ability to distinguish between high-risk and low-risk patients; we 
quantified discrimination and determined the cut-off values for the PNI divisions using Bayesian information criterion (BIC) [33]. A smaller BIC value indicates a more desirable stage division for predicting the outcome.

We used hazard ratios (HRs) and 95\% confidence intervals (CIs) to evaluate the association between PNI and CRC prognosis. To assess the relationship between PNI and clinicopathological features, odds ratios (ORs) and $95 \%$ CIs were used as effect measures. We used the method of Tierney [34] to estimate the HR and 95\% CI in the studies in which HR was not reported directly. Cochran's $\mathrm{Q}$ test and $\mathrm{I}^{2}$ statistics were used to evaluate heterogeneity. $\mathrm{I}^{2}>50 \%$ or/and $P<0.10$ indicated a statistically significant heterogeneity, which would allow the use of a random-effect model. Otherwise, a fixed-effect model was used.

Statistical analysis was performed using SPSS software version 20.0 (SPSS, Chicago, IL, USA) and STATA software version 12.0 (Stata Corporation, College Station, TX, USA). $P$-values $<0.05$ were considered statistically significant.

\section{ACKNOWLEDGMENTS}

This work was supported by National Science Foundation of China (81372549, 81372550), the Special Prophase Program for National Key Basic Research Program of China (No.2014CB560712) and Clinical Capability Construction Project for Liaoning Provincial Hospitals (LNCCC-A01-2014).

\section{CONFLICTS OF INTEREST}

The authors declare that they have no conflict of interest.

\section{REFERENCES}

1. Torre LA, Bray F, Siegel RL, Ferlay J, Lortet-Tieulent J and Jemal A. Global cancer statistics, 2012. CA Cancer J Clin. 2015; 65:87-108.

2. De Stefano A, Moretto R, Bucci L, Pepe S, Romano FJ, Cella AC, Attademo L, Rosanova M, De Falco S, Fiore G, Raimondo L, De Placido S and Carlomagno C. Adjuvant treatment for locally advanced rectal cancer patients after preoperative chemoradiotherapy: when, and for whom? Clinical colorectal cancer. 2014; 13:185-191.

3. Papamichael D, Audisio RA, Glimelius B, de Gramont A, Glynne-Jones R, Haller D, Kohne CH, Rostoft S, Lemmens V, Mitry E, Rutten H, Sargent D, Sastre J, Seymour M, Starling N, Van Cutsem E, et al. Treatment of colorectal cancer in older patients: International Society of Geriatric Oncology (SIOG) consensus recommendations 2013. Annals of oncology. 2015; 26:463-476.

4. Mantovani A, Allavena P, Sica A and Balkwill F. Cancer- related inflammation. Nature. 2008; 454:436-444.

5. Balkwill $\mathrm{F}$ and Mantovani $\mathrm{A}$. Inflammation and cancer: back to Virchow? Lancet. 2001; 357:539-545.

6. Schwegler I, von Holzen A, Gutzwiller JP, Schlumpf R, Muhlebach S and Stanga Z. Nutritional risk is a clinical predictor of postoperative mortality and morbidity in surgery for colorectal cancer. The British journal of surgery. 2010; 97:92-97.

7. Gupta D and Lis CG. Pretreatment serum albumin as a predictor of cancer survival: a systematic review of the epidemiological literature. Nutrition journal. 2010; 9:69.

8. Onodera T, Goseki N and Kosaki G. [Prognostic nutritional index in gastrointestinal surgery of malnourished cancer patients]. [Article in Japanese]. Nihon Geka Gakkai zasshi. 1984; 85:1001-1005.

9. Tabira Y, Okuma T, Kondo K, Yoshioka M, Mori T, Tanaka M, Nakano K and Kitamura N. Does neoadjuvant chemotherapy for carcinoma in the thoracic esophagus increase postoperative morbidity? The Japanese journal of thoracic and cardiovascular surgery. 1999; 47:361-367.

10. Sun $\mathrm{K}$, Chen $\mathrm{S}, \mathrm{Xu} \mathrm{J}, \mathrm{Li} \mathrm{G}$ and He Y. The prognostic significance of the prognostic nutritional index in cancer: a systematic review and meta-analysis. Journal of cancer research and clinical oncology. 2014; 140:1537-1549.

11. Chan AW, Chan SL, Wong GL, Wong VW, Chong CC, Lai PB, Chan HL and To KF. Prognostic Nutritional Index (PNI) Predicts Tumor Recurrence of Very Early/Early Stage Hepatocellular Carcinoma After Surgical Resection. Annals of surgical oncology. 2015; 22:4138-48.

12. Kanda M, Fujii T, Kodera Y, Nagai S, Takeda S and Nakao A. Nutritional predictors of postoperative outcome in pancreatic cancer. The British journal of surgery. 2011; 98:268-274.

13. Fu Y, Chen SW, Chen SQ, Ou-Yang D, Liu WW, Song M, Yang AK and Zhang Q. A Preoperative Nutritional Index for Predicting Cancer-Specific and Overall Survival in Chinese Patients With Laryngeal Cancer: A Retrospective Study. Medicine. 2016; 95:e2962.

14. Jeon HG, Choi DK, Sung HH, Jeong BC, Seo SI, Jeon SS, Choi HY and Lee HM. Preoperative Prognostic Nutritional Index is a Significant Predictor of Survival in Renal Cell Carcinoma Patients Undergoing Nephrectomy. Annals of surgical oncology. 2016; 23:321-7.

15. Migita K, Takayama T, Saeki K, Matsumoto S, Wakatsuki $\mathrm{K}$, Enomoto K, Tanaka T, Ito M, Kurumatani N and Nakajima Y. The prognostic nutritional index predicts longterm outcomes of gastric cancer patients independent of tumor stage. Annals of surgical oncology. 2013; 20:26472654.

16. Mohri Y, Inoue Y, Tanaka K, Hiro J, Uchida K and Kusunoki M. Prognostic nutritional index predicts postoperative outcome in colorectal cancer. World journal of surgery. 2013; 37:2688-2692.

17. Tokunaga R, Sakamoto Y, Nakagawa S, Miyamoto Y, 
Yoshida N, Oki E, Watanabe M and Baba H. Prognostic Nutritional Index Predicts Severe Complications, Recurrence, and Poor Prognosis in Patients With Colorectal Cancer Undergoing Primary Tumor Resection. Diseases of the colon and rectum. 2015; 58:1048-1057.

18. Liang L, Zhu J, Jia H, Huang L, Li D, Li Q and Li X. Predictive value of pretreatment lymphocyte count in stage II colorectal cancer and in high-risk patients treated with adjuvant chemotherapy. Oncotarget. 2016; 7:1014-1028. doi: 10.18632/oncotarget.5835.

19. Ray-Coquard I, Cropet C, Van Glabbeke M, Sebban C, Le Cesne A, Judson I, Tredan O, Verweij J, Biron P, Labidi I, Guastalla JP, Bachelot T, Perol D, Chabaud S, Hogendoorn PC, Cassier P, et al. Lymphopenia as a prognostic factor for overall survival in advanced carcinomas, sarcomas, and lymphomas. Cancer research. 2009; 69:5383-5391.

20. Artinyan A, Orcutt ST, Anaya DA, Richardson P, Chen GJ and Berger DH. Infectious postoperative complications decrease long-term survival in patients undergoing curative surgery for colorectal cancer: a study of 12,075 patients. Annals of surgery. 2015; 261:497-505.

21. Law WL, Choi HK, Lee YM and Ho JW. The impact of postoperative complications on long-term outcomes following curative resection for colorectal cancer. Annals of surgical oncology. 2007; 14:2559-2566.

22. Sakurai K, Ohira M, Tamura T, Toyokawa T, Amano R, Kubo N, Tanaka H, Muguruma K, Yashiro M, Maeda K and Hirakawa K. Predictive Potential of Preoperative Nutritional Status in Long-Term Outcome Projections for Patients with Gastric Cancer. Annals of surgical oncology. 2016; 23:525-33.

23. Song A, Eo W and Lee S. Comparison of selected inflammation-based prognostic markers in relapsed or refractory metastatic colorectal cancer patients. World journal of gastroenterology. 2015; 21:12410-12420.

24. Neal CP, Cairns V, Jones MJ, Masood MM, Nana GR, Mann CD, Garcea G and Dennison AR. Prognostic performance of inflammation-based prognostic indices in patients with resectable colorectal liver metastases. Medical oncology (Northwood, London, England). 2015; 32:144.

25. Jian-Hui C, Iskandar EA, Cai SI, Chen CQ, Wu H, Xu JB and He YL. Significance of Onodera's prognostic nutritional index in patients with colorectal cancer: a large cohort study in a single Chinese institution. Tumour biology. 2016; 37:3277-83.

26. Iseki $Y$, Shibutani M, Maeda K, Nagahara H, Ohtani H, Sugano K, Ikeya T, Muguruma K, Tanaka H, Toyokawa T, Sakurai K and Hirakawa K. Impact of the Preoperative Controlling Nutritional Status (CONUT) Score on the Survival after Curative Surgery for Colorectal Cancer. PloS one. 2015; 10:e0132488.

27. Ikeya T, Shibutani M, Maeda K, Sugano K, Nagahara H, Ohtani $\mathrm{H}$ and Hirakawa K. Maintenance of the nutritional prognostic index predicts survival in patients with unresectable metastatic colorectal cancer. Journal of cancer research and clinical oncology. 2015; 141:307-313.

28. Ihara K, Yamaguchi S, Shida Y, Ogata H, Domeki Y, Okamoto K, Nakajima M, Sasaki K, Tsuchioka T and Kato H. Poor nutritional status before and during chemotherapy leads to worse prognosis in unresectable advanced or recurrent colorectal cancer. International surgery. 2015.

29. Maeda K, Shibutani M, Otani H, Nagahara H, Sugano K, Ikeya T, Kubo N, Amano R, Kimura K, Muguruma K, Tanaka $\mathrm{H}$ and Hirakawa K. Low nutritional prognostic index correlates with poor survival in patients with stage IV colorectal cancer following palliative resection of the primary tumor. World journal of surgery. 2014; 38:12171222.

30. Nozoe T, Kohno M, Iguchi T, Mori E, Maeda T, Matsukuma A and Ezaki T. The prognostic nutritional index can be a prognostic indicator in colorectal carcinoma. Surgery today. 2012; 42:532-535.

31. Proctor MJ, Morrison DS, Talwar D, Balmer SM, Fletcher CD, O'Reilly DS, Foulis AK, Horgan PG and McMillan DC. A comparison of inflammation-based prognostic scores in patients with cancer. A Glasgow Inflammation Outcome Study. European journal of cancer (Oxford, England : 1990). 2011; 47:2633-2641.

32. Stang A. Critical evaluation of the Newcastle-Ottawa scale for the assessment of the quality of nonrandomized studies in meta-analyses. European journal of epidemiology. 2010; 25:603-605.

33. Volinsky CT and Raftery AE. Bayesian information criterion for censored survival models. Biometrics. 2000; $56: 256-262$.

34. Tierney JF, Stewart LA, Ghersi D, Burdett S and Sydes MR. Practical methods for incorporating summary time-toevent data into meta-analysis. Trials. 2007; 8:16. 